\title{
Estudos clínicos randomizados para registro de biossimilares trastuzumabe: uma revisão de escopo, Brasil, 2020
}

\author{
Randomized clinical trials to register biosimilar trastuzumab drugs: \\ a scoping review, Brazil, 2020
}

\author{
Tamiris Fazza Kelmer* iD \\ Mario Jorge Sobreira da Silva \\ Maely Peçanha Favero Retto
}

\section{RESUMO}

Introdução: 0 câncer de mama apresenta alta taxa de incidência no Brasil e no mundo e estima-se que cerca de $20 \%$ dos casos sejam classificados como Human Epidermal Growth Fator Receptor - tipo 2-positivo (HER2-positivo). Para tratamento desse tipo de câncer é indicado o uso de terapia-alvo, utilizando medicamentos biológicos, dentre eles, trastuzumabe. Por ser um medicamento considerado de alto custo, a entrada de seus biossimilares no mercado pode promover redução de custos aos serviços de saúde. Objetivo: Analisar os estudos clínicos de fase III de trastuzumabe biossimilares aprovados no Brasil até o ano de 2020. Método: Foi realizada uma revisão de escopo com estudos clínicos utilizados para o registro dos medicamentos biossimilares trastuzumabe no âmbito da Agência Nacional de Vigilância Sanitária (Anvisa). Os dados foram analisados quanto: i) aos protocolos de tratamento envolvidos nos estudos; ii) aos desfechos e características das populações investigadas; iii) ao perfil de segurança dos medicamentos biossimilares. Resultados: Foram selecionados, analisados e comparados seis estudos. Os estudos foram realizados com protocolos de tratamento, objetivos e medicamentos diferentes. A taxa de resposta completa foi o desfecho primário analisado na maioria dos estudos, seguido da resposta patológica completa. Em relação à população, os estudos envolveram a análise da população com intenção de tratar e/ou os pacientes que completaram o tratamento. Em todos os estudos, o perfil de segurança dos medicamentos biossimilares foi semelhante ao do medicamento de referência. Conclusões: Os estudos analisados foram capazes de demonstrar similaridade entre biossimilares e o medicamento de referência em relação à eficácia e à segurança, porém apresentaram diferenças em relação à metodologia utilizada, população e desfechos analisados.

PALAVRAS-CHAVE: Neoplasia de Mama; Trastuzumabe; Biossimilar

\section{ABSTRACT}

Introduction: Breast cancer has high incidence rates in Brazil and worldwide and it is estimated that about $20 \%$ of them are classified as Human Epidermal Growth Fator Receptor - tipo 2-positive (HER2-positive). For the treatment of this type of cancer, the use of targeted therapies is indicated, using biological drugs, among them, trastuzumab. As it is considered a high-cost drug, the entry of biosimilars into the market can reduce costs to health care services. Objective: To analyze the phase III clinical studies of biosimilar trastuzumab approved in Brazil until 2020. Methods: A scoping review was conducted with clinical trials used to register biosimilar trastuzumab drugs at Anvisa. The data were analyzed regarding: i) treatment protocols involved in the studies; ii) endpoints and investigated population features; iii) biosimilar drugs safety profile. Results: Six randomized clinical trials were selected, analyzed, and compared. The studies were carried out with different treatment protocols, endpoints and drugs. The complete response rate was analyzed in most studies, followed by the complete 
pathological response. Regarding the investigated population, the studies involved the analysis of the intention-to-treat population and/or per-protocol. In all studies, the biosimilar safety profile was similar to that of the reference drug. Conclusions: The analyzed studies were able to demonstrate similarity between biosimilars and the reference drug regarding safety and efficacy; however, they showed differences in their methodology, population and outcomes analyzed.

KEYWORDS: Breast Cancer; Trastuzumab; Biosimilar

\section{INTRODUÇÃO}

O câncer de mama é uma das maiores causas de mortalidade e morbidade em mulheres, com previsão de 66.280 novos casos, para cada ano do triênio 2020-2022. Desconsiderando casos de câncer de pele não melanoma, o câncer de mama feminina se destaca como o mais frequente em todas as regiões do Brasil ${ }^{1}$.

Estima-se que, em torno de $20 \%$ dos carcinomas de mama são caracterizados por amplificação e/ou superexpressão de Human Epidermal Growth Fator Receptor - tipo 2 (HER2), receptor transmembrana com atividade de tirosina-quinase. Esse subtipo de câncer apresenta pior prognóstico, caso não seja tratado da forma correta. Porém, a presença de HER2 aumenta as possibilidades terapêuticas, com a chamada terapia-alvo, que utiliza medicamentos que atuam especificamente neste receptor ${ }^{2}$.

Trastuzumabe é um anticorpo monoclonal, desenvolvido a partir da técnica de DNA recombinante. Age seletivamente no domínio extracelular da proteína do receptor HER2. Seus estudos demonstram que o medicamento inibe a proliferação de células tumorais humanas com superexpressão de HER2. Seu uso em combinação com quimioterapia tem impacto direto na sobrevida global das pacientes ${ }^{3,4}$.

O medicamento referência, Herceptin $®$, foi inicialmente aprovado para tratamento de câncer de mama metastático HER2-positivo nos Estados Unidos pela Food and Drug Administration (FDA) em 1998, e em 2000 pela Agência Europeia de Medicamentos (EMA). Logo depois, foi autorizado seu uso, pelas mesmas agências reguladoras, para tratamento adjuvante de câncer de mama HER2-positivo com comprometimento linfonodal e, em 2011, pela EMA, para terapia neoadjuvante para câncer de mama HER2-positivo inicial ${ }^{5}$.

No Brasil, o trastuzumabe foi incorporado ao Sistema Único de Saúde (SUS) no ano de 2012, por meio das Portarias da Secretaria de Ciência, Tecnologia, Inovação e Insumos Estratégicos em Saúde do Ministério da Saúde (SCTIE-MS) $n^{\circ} 18$ e n 19 , de 25 de julho, com indicação para câncer de mama HER2-positivo inicial e localmente avançado ${ }^{6,7}$.

Em 2017, após consulta pública e avaliação da Comissão Nacional de Incorporação de Tecnologias no SUS (Conitec), através da Portaria $n^{\circ} 29$, de 2 de agosto de 2017, trastuzumabe foi incorporado ao SUS para tratamento também de câncer de mama metastático em primeira linha ${ }^{8}$.

Desde o início dos anos 2000, medicamentos biológicos têm se tornado parte essencial no tratamento de câncer e outras doenças, porém, por apresentarem preços mais elevados, representam altos custos tanto para pacientes, como para os sistemas de saúde no geral. Para garantia de reembolso dos custos de pesquisa e desenvolvimento para aprovação desses medicamentos, até maio de 2021, era garantido um período de proteção, de patente, de em torno de 20 anos no Brasil, que poderia ser prorrogado. Com a aprovação da Ação Direta de Inconstitucionalidade $\mathrm{n}^{\circ} 5.529$, essa prorrogação não pode ser realizada e o prazo de vigência da patente de invenção voltou a ser de 20 anos e o de modelo de utilidade sete anos ${ }^{9}$. Uma vez que esse período é expirado, o registro, a produção e a comercialização de biossimilares se torna legalmente permitido ${ }^{10,11}$.

Biossimilares são produtos biológicos altamente similares aos de referência, que apresentam mínimas diferenças em seus componentes clinicamente inativos, sem possuir diferenças significativas em segurança, pureza ou potência, quando comparados ao produto de referência ${ }^{12}$.

No Brasil, a regulamentação em relação a produtos biológicos similares é feita pela Resolução da Diretoria Colegiada (RDC) $\mathrm{n}^{\circ} 55$, de 16 de dezembro de 2010, que define que, para registro, devem ser apresentados estudos de comparação entre o biossimilar e o produto biológico de referência que contenham informações suficientes para predizer que as diferenças detectadas nos seus atributos de qualidade não resultam em diferenças em sua segurança e eficácia ${ }^{13}$.

Como biossimilares não são considerados moléculas idênticas, mas similares, estudos de equivalência farmacêutica e bioequivalência utilizados para aprovação de medicamentos genéricos não podem ser utilizados para comprovar sua equivalência terapêutica. Desse modo, estudos clínicos comparativos entre biossimilar e medicamento de referência são necessários. Além disso, para que seja possível extrapolar os resultados para outras indicações aprovadas para o produto biológico comparado, é necessário que a população estudada seja representativa ${ }^{14,15}$.

Os estudos realizados para desenvolvimento de biossimilares se dão de forma diferente daqueles utilizados para produção de medicamentos inovadores. Enquanto no primeiro caso os ensaios clínicos randomizados são as fases mais demoradas e complexas, no segundo, as fases clínicas podem ser realizadas de forma mais rápida, desde que seja possível comprovar, através de extensos estudos pré-clínicos, que aquela molécula desenvolvida de fato é similar à de referência ${ }^{16}$. Esse processo é interessante para o cenário brasileiro, uma vez que o país parece estar mais preparado do ponto de vista da infraestrutura científica e tecnológica para realizar os testes pré-clínicos e clínicos de fase II e III. 
Dessa forma, os estudos com medicamentos biossimilares se iniciam por meio de análises em relação ao medicamento de referência, com o objetivo de entender suas características para que seja possível a produção de um produto similar. Essa etapa é extensa e envolve análises estruturais, físico-químicas e biológicas, para que o novo produto consiga ter qualidades semelhantes ${ }^{16}$.

Em seguida, para análises de comparabilidade em relação à farmacocinética, farmacodinâmica e toxicidade são realizados testes in vitro e in vivo, nas fases pré-clínicas. Para que os estudos possam dar seguimento, é importante que o medicamento testado tenha perfis semelhantes ao medicamento de referência ${ }^{17}$.

Após comprovada similaridade, estudos clínicos de comparabilidade entre os medicamentos devem ser realizados. Para isso, é necessária a seleção de uma população homogênea, com sensibilidade para a detecção de quaisquer diferenças que possam aparecer em relação aos medicamentos analisados. 0 desenho do estudo deve ser capaz de demonstrar perfis de eficácia e segurança semelhantes e, por isso, estudos de equivalência são recomendados ${ }^{18,19}$.

A entrada de medicamentos biossimilares no mercado possibilita a redução de custos pelos sistemas de saúde, aumentando o acesso à assistência em saúde em oncologia, o que é importante, uma vez que a incidência de câncer vem aumentando ao longo dos anos ${ }^{11}$.

Para registro de medicamentos biossimilares no Brasil, há a exigência de que pelo menos um estudo de fase III de comparação com o medicamento de referência seja realizado, porém a Agência Nacional de Vigilância Sanitária (Anvisa) não especifica quais metodologias devem ser utilizadas, fazendo com que os estudos apresentem desenhos diferentes ${ }^{13}$.

Dessa forma, o objetivo do estudo foi analisar e comparar os estudos clínicos randomizados de fase III dos medicamentos biossimilares trastuzumabe utilizados para aprovação de registro junto a Anvisa até o ano de 2020 com relação às metodologias utilizadas, tipos de população analisadas, desfechos e resultados de segurança.

\section{MÉTODO}

Foi realizada uma revisão de escopo com estudos clínicos utilizados para o registro dos medicamentos biossimilares trastuzumabe no âmbito da Anvisa.
Primeiramente, foi feita a identificação de trastuzumabe biossimilares com registro ativo no Brasil por meio da ferramenta de consulta disponibilizada no portal da Anvisa. A pesquisa foi realizada utilizando, no campo de busca, "trastuzumabe" como princípio ativo (https://consultas.anvisa.gov.br/\#/ medicamentos/q/?substancia=23119).

Os medicamentos registrados como biossimilares foram selecionados e foi feita uma análise a partir das bulas disponíveis para a identificação do nome do estudo clínico de fase III realizado para comparação entre biossimilar e medicamento de referência.

Posteriormente, foi feita uma pesquisa nas bases de dados MEDLINE e Web of Science, no mês de junho de 2020, utilizando os nomes dos estudos coletados, com o objetivo de seleção dos artigos e posterior leitura na íntegra e análise para comparação.

No caso de estudos que apresentaram mais de uma publicação, aquela considerada mais recente foi selecionada.

Foi elaborada, no programa Exce ${ }^{\oplus}$, uma ficha para extração dos dados principais coletados e informações relevantes de cada estudo: nome dos autores, ano de publicação, número de identificação do ensaio clínico, desenho do estudo, tipo de câncer tratado, número de pacientes randomizados (total e por braço de tratamento), protocolos de tratamento, desfechos e resultados de segurança.

Após a leitura dos artigos selecionados, a ficha foi preenchida e os dados coletados foram comparados. Os dados foram analisados quanto: i) aos protocolos de tratamento envolvidos nos estudos; ii) aos desfechos e características das populações investigadas; iii) ao perfil de segurança dos medicamentos biossimilares.

\section{RESULTADOS}

Foram selecionados seis estudos clínicos randomizados, com cinco biossimilares diferentes: MYL-14010, PF-05280014, SB3, ABP 980 e CT-P6. De uma forma geral, cada biossimilar apresentou um estudo, no qual a equivalência foi analisada. Apenas PF-05280014 apresentou dois estudos associados: um de análise de equivalência e um de não inferioridade ${ }^{19,20,21,22,23,24}$.

A Tabela 1 apresenta um resumo dos principais dados coletados de cada um dos estudos.

Tabela 1. Resumo dos ensaios clínicos randomizados de comparação entre trastuzumabe biossimilares e medicamento de referência.

\begin{tabular}{|c|c|c|c|c|c|c|c|}
\hline Biossimilar & Autor (ano) & Estudo & NCT & Patrocinador & Desenho & $\begin{array}{c}\mathrm{N} \text { randomizado } \\
\text { (Biossimilar/comparador) }\end{array}$ & $\begin{array}{l}\text { Tipo de } \\
\text { câncer }\end{array}$ \\
\hline MYL-14010 & Rugo et al. $(2017)^{20}$ & Heritage & NCT02472964 & Mylan & Equivalência & $500(249 / 251)$ & Metastático \\
\hline \multirow[b]{2}{*}{ PF-05280014 } & Pegram et al. $(2019)^{21}$ & Reflections B237-02 & NCT01989676 & Pfizer & Equivalência & 707 (352/355) & Metastático \\
\hline & $\begin{array}{l}\text { Lammers et al. } \\
\qquad(2018)^{22}\end{array}$ & Reflections B327-04 & NCT02187744 & Pfizer & $\begin{array}{c}\text { Não } \\
\text { inferioridade }\end{array}$ & $226(114 / 112)$ & Inicial \\
\hline SB3 & Pivot et al. $(2008)^{23}$ & SB3-G31-BC & NCT02149524 & Samsung & Equivalência & $875(437 / 438)$ & Inicial \\
\hline ABP 980 & $\begin{array}{l}\text { Von Minckwitz et al. } \\
\qquad(2018)^{24}\end{array}$ & Lilac & NCT01901146 & Amgen & Equivalência & $725(364 / 361)$ & Inicial \\
\hline CT-P6 & Esteva et al. (2019) ${ }^{25}$ & CT-P6 3.2 & NCT02162667 & Celltrion & Equivalência & $549(271 / 278)$ & Inicial \\
\hline
\end{tabular}

Fonte: Elaborada pelos autores, 2021.

NCT: Clinical Trial Number. 
O número de pacientes randomizados variou de 226 a 875 , com um total de 3.582 pacientes. 0 estudo Reflections B327-04 apresentou o menor número de pacientes, 226. Isso se deu devido à diferente metodologia aplicada ao estudo (estudo de não inferioridade) 20,21,22,23,24,25.

Dois estudos foram realizados com pacientes com câncer de mama metastático, nos quais foi analisada a associação do biossimilar com quimioterapia para uso paliativo $20,21,22,23,24,25$. Quatro estudos foram realizados com pacientes com câncer de mama inicial, analisando quimioterapia neoadjuvante e adjuvante, após cirurgia de remoção do tumor ${ }^{22,23,24,25}$.

Os efeitos de uma possível intercambialidade com o medicamento de referência foram avaliados em apenas um estudo, Lilac, que apresentou, em seu desenho, um switch, ou troca, na fase adjuvante, onde uma parte dos pacientes inicialmente randomizados para tratamento com o medicamento de referência trocaram seu tratamento para o biossimilar estudado, de forma aleatória ${ }^{24}$.

\section{Protocolos}

Os estudos fizeram uso de protocolos variados (Tabela 2). Os estudos que realizaram quimioterapia paliativa apresentaram protocolos semelhantes, uma vez que no estudo Reflections B237-02 foi utilizada associação de biossimilar e paclitaxel e, no Heritage, a combinação entre biossimilar e taxano também foi utilizada, porém a escolha do taxano (paclitaxel ou docetaxel) se deu a critério médico e da instituição responsável ${ }^{20,22,23,24,25}$.

Com relação aos estudos nos quais o tratamento foi realizado de forma adjuvante e neoadjuvante, é possível observar semeIhanças entre os protocolos utilizados nos estudos SB3-G31-BC e CT-P6 3.2, na etapa neoadjuvante, na qual os medicamentos antineoplásicos utilizados foram docetaxel, epirrubicina, 5-fluoruracila e ciclofosfamida. Já no estudo Lilac, o quimioterápico escolhido foi paclitaxel para uso de forma adjuvante, porém, no mesmo estudo, foi realizada uma quimioterapia inicial, com quatro ciclos de epirrubicina e ciclofosfamida anteriormente ao início do tratamento com o biossimilar. Nessa fase não houve nenhum tipo de análise em relação à eficácia ou à seguranç ${ }^{23,24,25}$. Nos estudos realizados com pacientes com câncer de mama inicial, após cirurgia, apenas o biossimilar foi utilizado na adjuvância.

A escolha do protocolo de tratamento foi utilizada com base em estudos prévios realizados com o medicamento referência, porém é possível perceber a grande variação entre os protocolos, onde para um mesmo fim, foram utilizados antineoplásicos de classes diferentes, dificultando a comparação entre eles.

\section{Desfechos e população analisada}

Na Tabela 3, estão descritos os desfechos e o tipo de população analisada em cada estudo.

Há certas diferenças em relação aos desfechos analisados em cada estudo. 0 mais utilizado, pathological complete response (PCR), se refere à taxa de resposta completa, ou seja, porcentagem de pacientes que atingiram resposta completa após o tratamento.
Outros desfechos similares foram utilizados em alguns estudos, breast pathological complete response (bpCR), em dois deles ${ }^{23,25}$ e total pathologic complete response (tpCR), em apenas um ${ }^{23}$. A diferença entre eles e o pCR é o fato de considerar, ou não, a presença de tumores axilares ou in situ ${ }^{25}$.

É possível destacar que esse tipo de desfecho somente foi possível nos estudos realizados com pacientes de câncer de mama inicial, uma vez que consideram remissão total da doença, o que não seria possível em pacientes com câncer metastático.

Para os dois estudos realizados com pacientes de câncer metastático, outros desfechos foram utilizados. 0 principal, objective response rate (ORR), utilizado em ambos, se refere à resposta parcial ou completa, sendo a resposta parcial definida pelo RECIST 1.1 como redução de pelo menos $30 \%$ das lesões em relação ao seu diâmetro ${ }^{20,21}$.

Outros desfechos utilizados para pacientes com câncer de mama metastático foram duração da resposta (DOR, duration of response), sobrevida livre de progressão (PFS, progression-free survival) e sobrevida global (OS, overall survival), calculados em meses.

Quanto à população analisada, é possível notar diferenças entre os estudos. A população chamada de intention-to-treat (ITT) se refere à população com intenção de tratar, ou seja, toda a população randomizada, independente de desistências ou perdas. 0 segundo tipo de população, per-protocol (PP), se refere apenas aos pacientes que completaram o tratamento $0^{20,22,23,24,25}$.

Tabela 2. Protocolos e medicamentos utilizados em cada estudo.

\begin{tabular}{|c|c|c|}
\hline Estudo & Protocolo & Quimioterápicos \\
\hline Heritage & Paliativo & Taxano \\
\hline Reflections B237-02 & Paliativo & Paclitaxel \\
\hline Reflections B327-04 & Neoadjuvante & Docetaxel e carboplatina \\
\hline SB3-G31-BC & Neoadjuvante & $\begin{array}{c}\text { Docetaxel, epirrubicina, } \\
\text { 5-fluoruracila e ciclofosfamida }\end{array}$ \\
\hline Lilac & Neoadjuvante & Paclitaxel \\
\hline CT-P6 3.2 & Neoadjuvante & $\begin{array}{c}\text { Docetaxel, epirrubicina, } \\
\text { 5-fluoruracila e ciclofosfamida }\end{array}$ \\
\hline
\end{tabular}

Fonte: Elaborada pelos autores, 2021.

Tabela 3. Desfechos e tipo de população analisada por estudo.

\begin{tabular}{lcc}
\hline Estudo & Desfecho primário & População analisada \\
\hline Heritage & ORR & ITT \\
Reflections B237-02 & ORR, DOR, PFS, OS & ITT/PP \\
Reflections B327-04 & pCR, ORR & PP \\
SB3-G31-BC & bPCR, tpCR, ORR, OS & ITT/PP \\
Lilac & pCR & PP \\
CT-P6 3.2 & pCR, bpCR & ITT/PP \\
\hline
\end{tabular}

Fonte: Elaborada pelos autores, 2021.

bpCR: breast pathological complete response (definido como ausência de carcinoma invasivo na mama, independente da presença de carcinoma ductal in situ ou envolvimento nodal); DOR: duration of response (duração da resposta); ITT: intention-to-treat (intenção de tratar); ORR: objective response rate (taxa de resposta objetiva); OS: overall survival (sobrevida global); pCR: pathologic complete response (definido como ausência de carcinoma invasivo na mama e linfonodos axilares, independentemente de carcinoma ductal in situ); PFS: progression-free survival (sobrevida livre de progressão); PP: per-protocol (por protocolo); tpCR: total pathologic complete response (definido como ausência de carcinoma invasivo e carcinoma in situ na mama e linfonodos axilares). 
A análise considerando ambos os tipos de população foi realizada em três estudos, enquanto três optaram por analisar somente um tipo, sendo que ITT foi escolhida em um estudo, e PP em outros dois. Considerando que as perdas e desvios de protocolo se dão numa parte relevante da população inicialmente randomizada, tais resultados podem apresentar divergências significativas ${ }^{20,21,22,23,24,25}$.

\section{Segurança}

A análise da segurança dos medicamentos biossimilares, em comparação ao medicamento referência, foi realizada baseada na ocorrência de eventos adversos (EA), inclusive aqueles considerados graves. A Tabela 4 apresenta um resumo dos dados coletados.

A ocorrência de EA, em todos os estudos, foi elevada e acometeu a maioria das pacientes estudadas.

Quanto à ocorrência de EA graves, ou seja, aqueles considerados de grau 3 ou mais, ou que necessitem de internação hospitalar, os números encontrados para cada estudo variaram, mas, de uma forma geral, acometeram menor número de pacientes.

Na maioria dos estudos, os resultados de segurança foram apresentados de uma forma geral, em números que englobavam a ocorrência de EA tanto na fase neoadjuvante quanto adjuvante (exceto estudos com protocolo paliativo). Porém o estudo Lilac apresentou tais dados considerando a ocorrência de cada tipo de EA em cada fase do estudo.

Os EA mais comuns foram, em grande parte, semelhantes entre os estudos, com destaque para alopecia, neutropenia e anemia. Os estudos Lilac e CT-P6 3.2 relataram outros, como infecções, reação infusional e rash, se diferenciando dos demais ${ }^{20,21,22,23,24,25}$.

Como trastuzumabe é um medicamento capaz de gerar uma cardiotoxicidade importante, os estudos realizaram análises específicas em relação à queda da fração de injeção de ventrículo esquerdo (FEVE), importante fator para monitorar tais efeitos adversos. Todos demonstraram resultados semelhantes entre o grupo que utilizou o medicamento biossimilar e o de referência ${ }^{20,21,22,23,24,25}$.
Em todos os estudos analisados, foi concluído que não há diferenças significativas em relação aos EA ocasionados pelo produto de referência e seus biossimilares, demonstrando que todos os tratamentos analisados são seguros $20,21,22,23,24,25$.

\section{DISCUSSÃO}

Assim como em agências regulatórias ao redor do mundo, como FDA e EMA, para aprovação de um medicamento biológico similar, a Anvisa, por meio da RDC $n^{\circ} 55 / 2010$, exige que pelo menos um estudo de fase III de comparação com o medicamento inovador seja apresentado, não especificando quais metodologias devem ser utilizadas e quais desfechos devem ser analisados ${ }^{13}$.

Essa normativa abre prerrogativa para que cada estudo seja realizado com metodologia distinta, o que pode gerar certas dificuldades de comparação entre os estudos de biossimilares, podendo interferir na decisão de intercambialidade entre eles ${ }^{13}$.

Como exposto, é possível perceber que, primeiramente, os estudos envolvem populações com perfis diferentes. É sabido, através de estudos anteriores realizados com o medicamento de referência, que o trastuzumabe é indicado para tratamento tanto de câncer de mama metastático como inicial. Porém, os estudos apresentados foram realizados considerando somente um tipo de tumor e apenas um biossimilar foi testado para ambas as indicações ${ }^{20,21,22,23,24,25}$.

A RDC $n^{\circ}$ 55/2010 permite que haja extrapolação de indicação, ou seja, uma vez que o medicamento foi comprovado eficaz para uma das indicações presentes na bula do medicamento referência, é possível extrapolar a indicação do biossimilar para as outras indicações presentes ${ }^{13}$.

Dessa forma, analisando a bula de cada um dos biossimilares e comparando-a com a bula do medicamento de referência, é possível afirmar que houve extrapolação de indicação para todos os biossimilares. Apesar de os estudos terem sido realizados, em sua maioria, com apenas um tipo de câncer de mama, todos eles

Tabela 4. Ocorrência de eventos adversos por estudo.

\begin{tabular}{|c|c|c|c|c|c|c|c|c|}
\hline \multirow[b]{2}{*}{ Estudo } & \multicolumn{4}{|c|}{ Ocorrência de EA } & \multicolumn{3}{|c|}{ EA grave } & \multirow[b]{2}{*}{ Principais EA } \\
\hline & $\begin{array}{l}\mathscr{N} \\
\stackrel{\varpi}{\leftarrow}\end{array}$ & 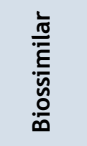 & 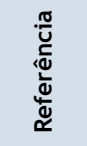 & 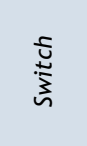 & 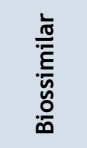 & 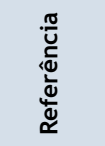 & 志 & \\
\hline Heritage & & $96,8 \%$ & $94,7 \%$ & & $38,1 \%$ & $36,2 \%$ & & Alopecia, neutropenia \\
\hline Reflections B237-02 & & $96,6 \%$ & $96,0 \%$ & & $15,2 \%$ & $15,9 \%$ & & Alopecia, anemia, neutropenia \\
\hline Reflections B327-04 & Todas* & $96,5 \%$ & $94,6 \%$ & & $6,2 \%$ & $5,4 \%$ & & Alopecia, anemia, neutropenia \\
\hline SB3-G31-BC & Todas* & $97,5 \%$ & $96,1 \%$ & & $12,8 \%$ & $13,2 \%$ & & Alopecia, neutropenia \\
\hline \multirow{2}{*}{ Lilac } & NA & $80,2 \%$ & $79,5 \%$ & & $4,9 \%$ & $1,4 \%$ & & \multirow{2}{*}{ Neutropenia, infecções, reação infusional } \\
\hline & A & $57,6 \%$ & $52,0 \%$ & $57,3 \%$ & $4,0 \%$ & $2,3 \%$ & $2,3 \%$ & \\
\hline CT-P6 3.2 & Todas & $97,0 \%$ & $95,3 \%$ & & $7,4 \%$ & $11,9 \%$ & & Neutropenia, anemia, rash \\
\hline
\end{tabular}

Fonte: Elaborada pelos autores, 2021.

* Todas: adjuvante e neoadjuvante; NA: neoadjuvante; A: adjuvante; EA: evento adverso. 
apresentam as mesmas indicações: câncer de mama metastático (em monoterapia ou em combinação com paclitaxel ou docetaxel) e câncer de mama inicial (após cirurgia, quimioterapia e radioterapia ou em combinação com quimioterapia). Além disso, nenhum dos estudos realizou análises com pacientes com câncer gástrico avançado, mas essa indicação está presente em suas bulas, demonstrando que houve extrapolação para todas as indicações do medicamento de referência ${ }^{3,26,27,28,29,30}$.

Outro fator importante a destacar é que a escolha de protocolos também apresentou grande variação. Enquanto os estudos propostos para tratamento paliativo utilizaram protocolo semethante, os estudos realizados com finalidade neoadjuvante apresentaram divergências com relação à escolha dos antineoplásicos utilizados, tanto em relação à quantidade quanto às classes.

Tais escolhas se deram baseadas em estudos prévios com o medicamento de referência, mostrando que, de fato, para esses protocolos, os biossimilares são eficazes, porém, na prática, em cada centro de tratamento um tipo de protocolo é utilizado. Se levarmos em consideração o contexto da saúde pública, essa escolha pode ser bem limitada, pois deve-se levar em consideração os medicamentos disponíveis em cada instituição ${ }^{31}$.

Apenas um estudo apresentou análise em relação à intercambialidade entre o medicamento biossimilar e o de referência. Esse tipo de análise é interessante, pois é capaz de demonstrar, na prática, os efeitos que uma possível intercambialidade pode causar ${ }^{24}$. Como isso é algo constante na prática clínica, é importante ser possível demonstrar, por meio de análises detalhadas, que não é capaz de gerar perdas para o paciente em relação ao seu tratamento. Por isso, seria interessante que estudos futuros se preocupassem com esse fato.

Além disso, a necessidade desse tipo de estudo para intercambialidade depende da agência regulatória de cada país. A FDA, por exemplo, exige que estudos de switch sejam realizados para que a intercambialidade seja permitida ${ }^{32}$.

Em relação aos desfechos analisados, as diferenças entre os estudos são justificáveis devido à finalidade do tratamento proposto.

Os resultados apresentados pelos estudos selecionados foram satisfatórios, pois demonstraram que, em comparação ao medicamento de referência, há similaridade entre eles.

A metodologia para análise da segurança utilizada pelos estudos foi semelhante e foi capaz de demonstrar que, de uma forma geral, muitos pacientes que fazem tratamento com trastuzumabe apresentam EA, inclusive aqueles considerados graves. Porém, esses efeitos, em sua maioria, são bem tolerados e passíveis de serem tratados. As análises realizadas demonstraram perfil de segurança similar entre os grupos dos estudos, confirmando que o tratamento com os biossimilares é tão seguro quanto o tratamento com o medicamento de referência ${ }^{20,21,22,23,24,25}$.

No entanto, o presente estudo apresenta certas limitações, como a utilização de dados coletados em estudos utilizados para registro, sendo que alguns deles ainda se encontram em andamento. Além disso, cada estudo de fase III realizado pode gerar outras publicações, com dados interessantes, que não foram analisados. Como pesquisas futuras, sugere-se a realização de estudos que analisem o efeito da introdução dos biossimilares na redução de preços e a realização de estudos clínicos comparativos entre biossimilares e medicamentos de referência que possam contribuir para a obtenção de um maior conjunto de dados sobre efetividade e segurança dessas tecnologias.

\section{CONCLUSÕES}

Os estudos clínicos randomizados de fase III realizados com trastuzumabe biossimilares foram capazes de comprovar sua equivalência ao medicamento de referência, tanto em relação à sua eficácia, como em relação à segurança.

Porém, é possível destacar que há grandes diferenças entre os estudos, tanto no que diz respeito às características da população randomizada como em relação aos protocolos e desfechos clínicos analisados. Por esse motivo, há certa dificuldade em comparar resultados.

Para mudança desse cenário, recomenda-se que a Anvisa proponha revisão da normativa vigente ou emita notas técnicas que explicitem as metodologias que devem ser utilizadas, o tamanho da população a ser incluída e os desfechos que devem ser analisados nos estudos clínicos com medicamentos biossimilares.

Atualmente não há estudos de comparabilidade entre biossimilares, não sendo possível comprovar que haja possibilidade de intercambialidade entre eles. Dessa forma, torna-se importante que esses estudos sejam realizados futuramente para que a intercambialidade possa ser realizada, sem quaisquer prejuízos ao tratamento.

Por fim, destaca-se que a introdução dos biossimilares no mercado possibilitou a comercialização de um medicamento fundamental no tratamento de câncer de mama para a população, contribuindo, assim, para que mais pacientes tenham acesso a um tratamento de qualidade.

\section{REFERÊNCIAS}

1. Instituto Nacional de Câncer José Alencar Gomes da Silva - INCA. Estimativa 2020: incidência de câncer no Brasil.

Rio de Janeiro: Instituto Nacional de Câncer José Alencar Gomes da Silva; 2019.

2. Fabi A, Malaquti P, Vari S, Cognetti F. First-line therapy in HER2 positive metastatic breast cancer: is the mosaic fully completed or are we missing additional pieces? J Exp Clin Cancer Res. 2016;35:1-11. https://doi.org/10.1186/s13046-016-0380-5

3. Roche Químicos e Farmacêuticos. Herceptin $®$ : trastuzumabe. São Paulo: Roche Químicos e Farmacêuticos; 2020[acesso 18 set 2020]. Disponível em: https://consultas.anvisa.gov.br/\#/ medicamentos/250000233539912/?nomeProduto=HERCEPTIN 
4. Molina MA, Codony-Servat J, Albanell J, Rojo F, Arribas J, Baselga J. Trastuzumab (herceptin), a humanized anti-HER2 receptor monoclonal antibody, inhibits basal and activated HER2 ectodomain cleavage in breast cancer cells. Cancer Res. 2001;61(12):4744-9.

5. Cargnin S, Shin JI, Genazzani AA, Nottegar A, Terrazzino S. Comparative efficacy and safety of trastuzumab biosimilars to the reference drug: a systematic review and meta-analysis of randomized clinical trials. Cancer Chemother Pharmacol. 2020;86(5):577-88. https://doi.org/10.1007/s00280-020-04156-3

6. Ministério da Saúde (BR). Portaria $N^{\circ} 18$, de 25 de julho de 2012. Torna pública a decisão de incorporar o medicamento trastuzumabe no Sistema Único de Saúde (SUS) para o tratamento do câncer de mama localmente avançado. Diário Oficial União. 26 jul 2012.

7. Ministério da Saúde (BR). Portaria $N^{\circ} 19$, de 25 de julho de 2012. Torna pública a decisão de incorporar o medicamento trastuzumabe no Sistema Único de Saúde (SUS) para o tratamento do câncer de mama inicial. Diário Oficial União. 26 jul 2012.

8. Ministério da Saúde (BR). Portaria $N^{\circ} 29$, de 2 de agosto de 2017. Torna pública a decisão de incorporar o trastuzumabe para o tratamento do câncer de mama HER2-positivo metastático em primeira linha de tratamento, conforme protocolo clínico e diretrizes terapêuticas do Ministério da Saúde, no âmbito do Sistema Único de Saúde - SUS. Diário Oficial União. 3 ago 2017.

9. Supremo Tribunal Federal - STF. Ação direta de inconstitucionalidade $N^{\circ} 5.529$, de 13 de maio de 2021. Torna pública a decisão de extinguir o dispositivo previsto no parágrafo único do artigo 40 da lei № 9.279/1996 (lei da propriedade industrial, LPI). Diário Oficial União. 14 maio 2021.

10. Paranhos J, Mercadante E, Hasenclever L. O custo da extensão da vigência de patentes de medicamentos para o Sistema Único de Saúde. Cad Saúde Pública. 2020;36(11):1-13. https://doi.org/10.1590/0102-311X00169719

11. Hübel K, Kron F, Lux MP. Biosimilars in oncology: effects on economy and therapeutic innovations. Eur J Cancer. 2020;139:10-9. https://doi.org/10.1016/j.ejca.2020.07.037

12. Blackwell K, Gligorov J, Jacobs I, Twelves C. The global need for a trastuzumab biosimilar for patients with HER2-positive breast cancer. Clin Breast Cancer. 2018;18(2):95-113. https://doi.org/0.1016/j.clbc.2018.01.006

13. Agência Nacional Vigilância Sanitária - Anvisa. Resolução RDC No 55, de 16 de dezembro de 2010. Dispõe sobre o registro de produtos biológicos novos e produtos biológicos e dá outras providências. Diário Oficial União. 17 dez 2010.

14. Ferreira Neto PTP, Nunes PHC, Vargas MA. Intercambialidade de produtos biológicos no Sistema Único de Saúde (SUS): principais desafios regulatórios. Cad Saúde Pública. 2019;35(10):1-15. https://doi.org/10.1590/0102-311X00053519

15. Fernandes GS, Sternberg C, Lopes G, Chammas R, Gifoni MAC, Gil RA et al. The use of biosimilar medicines in oncology: position statement of the brazilian society of clinical oncology (SBOC). Braz J Med Biol Res. 2018;51(3):1-7. https://doi.org/10.1590/1414-431X20177214

16. O'Callaghan J, Barry SP, Bermingham M, Morris $J M$, Griffin BT. Regulation of biosimilar medicines and current perspectives on interchangeability and policy. Eur J Clin Pharmacol. 2019;75(1):1-11. https://doi.org/10.1007/s00228-018-2542-1

17. Bui LA, Hurst $S$, Finch GL, Ingram B, Jacobs IA, Kirchhoff $\mathrm{CF}$ et al. Key considerations in the preclinical development of biosimilars. Drug Discov Today. 2015;20(Supl.):3-15. https://doi.org/10.1016/j.drudis.2015.03.011

18. Thill M. Biosimilar trastuzumab in clinical trials: differences or not? Breast Care. 2019;14(1):17-22. https://doi.org/10.1159/000496503

19. Weise M, Bielsky MC, De Smet K, Ehmann F, Ekman $\mathrm{N}$, Giezen TJ et al. Biosimilars: what clinicians should know. Blood. 2012;120(26):5111-7. https://doi.org/10.1182/blood-2012-04-425744

20. Rugo HS, Barve A, Waller CF, Hernandez-Bronchud M, Herson $\mathrm{J}$, Yuan $\mathrm{J}$ et al. Effect of a proposed trastuzumab biosimilar compared with trastuzumab on overall response rate in patients with ERBB2 (HER2): positive metastatic breast cancer: a randomized clinical trial. JAMA. 2017;317(1):37-47. https://doi.org/10.1001/jama.2016.18305

21. Pegram MD, Bondarenko I, Zorzetto MMC, Hingmire S, Iwase H, Krivorotko PV et al. PF-05280014 (a trastuzumab biosimilar) plus paclitaxel compared with reference trastuzumab plus paclitaxel for HER2-positive metastatic breast cancer: a randomised, double-blind study. Br J Cancer. 2019;120(2):172-82. https://doi.org/10.1038/s41416-018-0340-2

22. Lammers PE, Dank M, Masetti R, Abbas R, Hilton F, Coppola J et al. Neoadjuvant PF-05280014 (a potential trastuzumab biosimilar) versus trastuzumab for operable HER2+ breast cancer. Br J Cancer. 2018;119(3):266-73. https://doi.org/10.1038/s41416-018-0147-1

23. Pivot X, Bondarenko I, Nowecki Z, Dvorkin M, Trishkina E, Ahn JH et al. A phase III study comparing SB3 (a proposed trastuzumab biosimilar) and trastuzumab reference product in HER2-positive early breast cancer treated with neoadjuvant-adjuvant treatment: final safety, immunogenicity and survival results. Eur J Cancer. 2018;93:19-27. https://doi.org/10.1016/j.ejca.2018.01.072

24. von Minckwitz G, Colleoni M, Kolberg H-C, Morales $S$, Santi $P$, Tomasevic $Z$ et al. Efficacy and safety of ABP 980 compared with reference trastuzumab in women with HER2-positive early breast cancer (LILAC study): a randomised, double-blind, phase 3 trial. Lancet Oncol. 2018;19(7):987-98. https://doi.org/10.1016/S1470-2045(18)30241-9

25. Esteva FJ, Baranau YV, Baryash V, Manikhas A, Moiseyenko $V$, Dzagnidze $G$ et al. Efficacy and safety of CT-P6 versus reference trastuzumab in HER2-positive early breast cancer: updated results of a randomised phase 3 trial. Cancer Chemother Pharmacol. 2019;84(4):839-47. https://doi.org/10.1007/s00280-019-03920-4 
26. Celltrion Healthcare Distribuicao de Produtos Farmaceuticos do Brasil. Herzuma ${ }^{\circ}$ : trastuzumabe. São Paulo: Celltrion Healthcare Distribuicao de Produtos Farmaceuticos do Brasil; 2020[acesso 24 set 2020]. Disponível em: https: / consultas. anvisa.gov.br/\#/medicamentos/25351708654201326/? nomeProduto=herzuma

27. Amgen Biotecnologia do Brasil. Kanjinti®: trastuzumabe. São Paulo: Amgen Biotecnologia do Brasil; 2020[acesso 24 set 2020]. Disponível em: https://consultas.anvisa.gov.br/\#/ medicamentos $/ 25351557932201727 /$ ?nomeProduto=kanjinti

28. Samsung Bioepis Brasil Pharmaceutical. Ontruzant $₫$ : trastuzumabe. Itapevi: Samsung Bioepis; 2020[acesso 25 set 2020]. Disponível em: https://consultas.anvisa.gov.br/\#/ medicamentos/25351083624201832/ ?nomeProduto=ontruzant
29. Wyeth Indústria Farmacêutica. Trazimera®: trastuzumabe. São Paulo: Wyeth Indústria Farmacêutica; 2020[acesso 21 set 2020]. Disponível em: https://consultas.anvisa.gov. $\mathrm{br} / \# /$ medicamentos/25351367633201883/ ?nomeProduto=trazimera

30. Libbs Farmacêutica. Zedora®: trastuzumabe. São Paulo: Libbs Farmacêutica; 2019[acesso 18 set 2020]. Disponível em: https://consultas.anvisa.gov.br/\#/ medicamentos $/ 25351595780201605 /$ ?nomeProduto=zedora

31. Kaliks RA, Matos TF, Silva VA, Barros LH. Diferenças no tratamento sistêmico do câncer no Brasil: meu SUS é diferente do teu SUS. Braz J Oncol. 2017;13(44)1-12.

32. US Food and Drug Administration - FDA. Considerations in demonstrating interchangeability with a reference product guidance for industry. Rockville: US Food and Drug Administration; 2019.

Contribuição dos Autores

Kelmer TF - Concepção, planejamento (desenho do estudo), aquisição, análise, interpretação dos dados e redação do trabalho. Silva MJS - Análise, interpretação dos dados e redação do trabalho. Retto MPF - Concepção, planejamento (desenho do estudo), análise, interpretação dos dados e redação do trabalho. Todos os autores aprovaram a versão final do trabalho.

Conflito de Interesse

Os autores informam não haver qualquer potencial conflito de interesse com pares e instituições, políticos ou financeiros deste estudo.

Licença CC BY-NC atribuição não comercial. Com essa licença é permitido acessar, baixar (download), copiar, imprimir, compartilhar, reutilizar e distribuir os artigos, desde que para uso não comercial e com a citação da fonte, conferindo os devidos créditos de autoria e menção à Visa em Debate. Nesses casos, nenhuma permissão é necessária por parte dos autores ou dos editores. 